for domestic work and caretaking work. Animal husbandry was divided more evenly between males and females with their relative work contributions of $56 \%$ and $44 \%$, respectively. Animal husbandry-related injuries were divided correspondingly between the genders (males 57\% and females 43\%) but all other types of injuries occurred mostly to males.

Conclusions While many studies have shown that male farmers have a much greater risk of injury than females, our study shows that given equal work time, the risk of injury was also equal. Therefore, rather than a risk factor, gender is an indicator of different work exposures in farming. This finding suggests that targeting interventions to males is still appropriate, but it is more important to target the underlying hazardous work tasks and exposures as they increase the injury risk of both males and females.

\section{USING THE SOCIO-ECOLOGIC MODEL AS A GUIDE FOR AGRICULTURAL SAFETY INTERVENTIONS}

Barbara C Lee, Marsha Salzwedel, Bryan Weichelt, Casper Bendixsen. National Children's Centre for Rural and Agricultural Health and Safety, Marshfield, WI, USA

\subsection{6/injuryprev-2016-042156.363}

Background Agriculture is a dangerous industry that allows children in the worksite. Each day about 38 children are seriously injured on farms. The culture of agriculture resists advice from outsiders. Farm operators/parents prefer receiving information from familiar sources, not safety professionals. The Socio-ecologic Model (SEM) demonstrates how spheres of increasing influence have higher degrees of impact on individual behaviour.

Methods We modified the SEM to have farm children as the focal point, with adults/parents the first line of protection. Increasing spheres of influence are family and friends, followed by the community, organisations, and businesses. Public policy has the greatest influence but in agriculture rarely applies. Key farm business contacts are property and liability insurance providers. We conducted a study to assess if and how insurers could influence farmers regarding childhood farm safety. Surveys were distributed at a farm risk management training with 96 (55\%) insurers responding.

Results Agricultural insurers reported 55\% currently send general safety information and 38\% provide financial support of safety events. Regarding child-specific information, 79\% want to increase their focus on children and $63 \%$ need guidance for this. Furthermore, $76 \%$ believe customers would be pleased if they added a focus on childhood farm safety. Using these results, an illustrated report was sent to 150 agricultural insurance companies. It highlighted safety topics of concern, including ATVs and children operating tractors. Details were offered on insurance providers' strategies, such as co-branding farm safety posters, updating farm policies on young workers, and website links to and evidence-based programs.

Conclusions This new approach may influence farmers to protect children from preventable injuries. The project impact, including numbers and types of actions taken by insurance companies, will be shared at the Safety 2016 World Conference.

\section{DISABLING FARM INJURIES AND SAFETY INTERVENTIONS: AN INDIAN STUDY}

Adarsh Kumar, JK Singh. Indian Agricultural Research Institute, New Delhi, India

\subsection{6/injuryprev-2016-042156.364}

Background 260 million perons are associated with farm activities in India. Aaccording to Census 2011, a total of 26.8 million persons are disable. There is an increase of 5 million in last 10 years, indicating an addition of 1400 disabled individual every day. In agricultural "acquired disability" because of unsafe farm machine injuries is an added dimension to rural disability.

Methods: Compensation data of agricultural injury victims (156 cases) were collected from the Sonipat District, Haryana, India to assess kind of injuries and disabilities along with machines associated for the years 2008 to 2014. The most common machines associated with disabling injury was fodder cutter, safety interventions were developed and retrofitted in 50 households and feedback was collected.

Results Total bodily injuries were 105 out of 156 cases, out of which 84 cases $(80 \%)$ pertains to amputation of fingers, 13 cases involved and wrist (12\%), 5 cases (5\%) had leg injury, one person (1\%) injured his eye and four cases (4\%) injuries sustained on upper body. Machines associated with injuries were chaff cutters (74 cases), electric motors (20 cases), tube wells (6 cases), threshers (12 cases), tractor/trolley (9 cases), harrows ( 2 cases), and one each of reaper, sickle, winnower, and seed drill. The causal factor associated with fodder cutter injuries were; accessibility of children to machine, get injured while playfully interacting with this machine; hands injuries while feeding the fodder as entrapped in the rollers; diverted attention; loose clothes, get entangles in the gears and belt.

Conclusions Simple safety gadgets consisting of blade guard, flywheel lock, warning roller were developed and retrofitted in rural households and feedback suggested that it prevents injuries but some farmers of the opinion that warning roller hinders feeding of fodder quickly.

\section{BATTLEFIELD TO FARM FIELD: RISK PERCEPTIONS OF US MILITARY VETERANS TRANSITIONING INTO AGRICULTURE}

Casper G Bendixsen, Kathrine Barnes, Bryan Weichelt, Jeff VanWormer, Matthew C Keifer Marshfield Clinic Research Foundation, USA.

\subsection{6/injuryprev-2016-042156.365}

Background Prompted by wars in the past two decades largely in the Middle East, the United States veteran population in the United States often struggles with unemployment. America is increasingly occupied by the mental health ramifications of deployment. Domestically, the United States grapples with securing a healthy and abundant food source to support a large food insecure population in the midst of decreases in the number of farms and increasing food imports. The veteran-to-farmer (V2F) movement converges within these two concerns to provide jobs and potential therapeutic benefits to veterans through food production.

Methods The project combines a grounded theory approach with epidemiology to understand a broad range of implications for the V2F movement. Grounded theory will be utilised to conduct semi-structured interviews and participant-observation to explore 